\title{
Comparison of $\mathrm{Y}_{2} \mathrm{Ba}_{4} \mathrm{CuBiO}_{\mathrm{y}}$ Nanoparticles with $\mathrm{CeO}_{2}$ Doping on the Levitation Force of Single Domain YBCO Bulk Superconductor by TSIG Process
}

\author{
Miao Wang1* Xu Yang2, Xuan Wang³, Xiaomei Wang1, Ming Zhang1, Dapeng Hao1 \\ ${ }^{1}$ School of Science, Xi'an Aeronautical University, Xi'an, China \\ ${ }^{2}$ School of Electronic Engineering, Xi'an Aeronautical University, Xi'an, China \\ ${ }^{3}$ School of Material Engineering, Xi'an Aeronautical University, Xi'an, China \\ Email: *cwnanmao@126.com
}

How to cite this paper: Wang, M., Yang, X., Wang, X., Wang, X.M., Zhang, M. and Hao, D.P. (2018) Comparison of $\mathrm{Y}_{2} \mathrm{Ba}_{4} \mathrm{CuBiO}_{\mathrm{y}}$ Nanoparticles with $\mathrm{CeO}_{2}$ Doping on the Levitation Force of Single Domain YBCO Bulk Superconductor by TSIG Process. Journal of Materials Science and Chemical Engineering, 6, 90-98.

https://doi.org/10.4236/msce.2018.61010

Received: December 26, 2017

Accepted: January 26, 2018

Published: January 29, 2018

Copyright $\odot 2018$ by authors and Scientific Research Publishing Inc. This work is licensed under the Creative Commons Attribution International License (CC BY 4.0).

http://creativecommons.org/licenses/by/4.0/

\begin{abstract}
The top-seeded infiltration and growth process (TSIG) is very effective method for the preparation of $\mathrm{YBa}_{2} \mathrm{Cu}_{3} \mathrm{O}_{7-\mathrm{x}}$ (YBCO) bulk superconductors. In order to improve the levitation force of the samples, a series of single domain YBCO bulk superconductors with different ratios of nanoscale $\mathrm{Y}_{2} \mathrm{Ba}_{4} \mathrm{CuBiO}_{y}$ (YBi2411) inclusions in the solid phase pellet is successfully fabricated by the TSIG technique on the basis of previous research. In the present work, the results of YBCO bulk superconductors with YBi2411 and $\mathrm{CeO}_{2}(1 \mathrm{wt} \%)$ codoping system indicate that, the optimum doping of YBi2411 is $2 \mathrm{wt} \%$, the size of Y211 particles is reduced compared with the samples without $\mathrm{CeO}_{2}$ doping; the largest levitation force is about $15 \mathrm{~N}$ obtained in the samples with optimum YBi2411, which is about two times higher than that of the sample without $\mathrm{CeO}_{2}$ doping. The results are very helpful for the fabrication of high quality single domain $\mathrm{YBCO}$ bulk superconductors.
\end{abstract}

\section{Keywords}

Nanoparticles, YBCO Bulk Superconductor, TSIG Process

\section{Introduction}

High temperature superconductor (HTS) has been one of the international hotspots and impotent research contents all over the world [1]. Because of the high critical temperature, strong trapped flux density, large magnet levitation force and stable suspension characteristics, the single domain YBCO superconductors 
have significant potential for various applications, such as high field permanent magnets, magnetic bearing, flywheel, levitated transportation systems, motors, generators, and maglev systems, etc. [2]. It is well known that the top-seeded melt texture growth (TSMTG) method is widely used to fabricate bulk superconductors with high critical current densities $\left(J_{c}\right)$ at $77 \mathrm{~K}$ [3]. However, conventional melt processes based on the peritectic reaction have some frequent problems, such as existence of defect, the loss of liquid during peritectic decomposition, distortion, and shrinkage of the final samples. In order to solve these problems, a new technique based on the top-seeded infiltration and growth process (TSIG) has been developed by various laboratories in recent years [4]-[9]. Furthermore, the particles of $\mathrm{Y}_{2} \mathrm{BaCuO}_{5}(\mathrm{Y} 211)$ are an effective flux pinning center for single domain YBCO bulk superconductors, but the particle size is a little larger, from several micrometers to more than ten micrometer range. It is difficult to reduce the Y211 particle to the size less than $1 \mu \mathrm{m}$ and improve the flux pinning force further, even if Pt is added to reduce the Y211 particle [1] [10] [11] [12] [13]. So effective artificial pinning centers have to be introduced to improve $\mathrm{J}_{\mathrm{c}}$ of these materials [14]. It is found that a new kind of nanoscale inclusions $(\mathrm{RE})_{2} \mathrm{Ba}_{4} \mathrm{CuMO}_{\mathrm{y}}$ (where $\mathrm{RE}=$ rare earth, and $\mathrm{M}=\mathrm{Bi}, \mathrm{Ta}, \mathrm{W}, \mathrm{Mo}, \mathrm{Zr}, \mathrm{Hf}$, $\mathrm{Ag}, \mathrm{Sb}, \ldots)$ has been successfully synthesized and introduced into YBCO bulk superconductor by TSMTG, the result shows that the nanoscale $(\mathrm{RE})_{2} \mathrm{Ba}_{4} \mathrm{CuMO}_{\mathrm{y}}$ particles can work as effective flux pinning centers to improve $\mathrm{J}_{c}$ of the $\mathrm{YBCO}$ bulk superconductor [15].

In order to improve the levitation force of the samples, a series of single domain YBCO bulk superconductors with different ratios of nanoscale $\mathrm{Y}_{2} \mathrm{Ba}_{4} \mathrm{CuBiO}_{y}$ (YBi2411) inclusions in the solid phase pellet is successfully fabricated by the TSIG technique on the basis of previous research [16]. In this paper, we report the synthesis of nanoscale inclusion YBi2411 phase powder and, the growth morphology, microstructure and levitation force of the YBCO bulks. And at the same time, the effect of $1 \mathrm{wt} \% \mathrm{CeO}_{2}$ additions on the levitation force of $\mathrm{YBCO}$ bulks has also been researched, which is necessary for the fabrication of the high performance single domain YBCO bulk superconductors.

\section{Experimental}

The precursor powders of $\mathrm{Y} 211, \mathrm{YBa}_{2} \mathrm{Cu}_{3} \mathrm{O}_{7-\delta}(\mathrm{Y} 123)$ and $\mathrm{BaCuO}_{2}(\mathrm{Y} 011)$ were fabricated successfully by solid state synthesis. $\mathrm{YBi} 2411$ powder was synthesized according to following reaction formula:

$$
2 \mathrm{Y}_{2} \mathrm{O}_{3}+8 \mathrm{BaCO}_{3}+2 \mathrm{CuO}+\mathrm{Bi}_{2} \mathrm{O}_{3} \rightarrow 2 \mathrm{Y}_{2} \mathrm{Ba}_{4} \mathrm{CuBiO}_{y}+8 \mathrm{CO}_{2} \uparrow
$$

$\mathrm{Y}_{2} \mathrm{O}_{3}, \mathrm{BaCO}_{3}, \mathrm{CuO}$ and $\mathrm{Bi}_{2} \mathrm{O}_{3}$ powders (purity 99.9\%) were weighed and mixed in required molar ratio of $\mathrm{Y}: \mathrm{Cu}: \mathrm{Ba}: \mathrm{Bi}=2: 4: 1: 1$, and milled by a ball milling machine for 3 hours, then sintered at temperatures about $955^{\circ} \mathrm{C}$ for 3 times with interval grinding. X-ray diffraction (XRD) experiments were done to identify the purity of the YBi2411 phase. The XRD pattern of the sintered YBi2411 powder is shown in Figure 1. As we can see from this figure, there is no other 


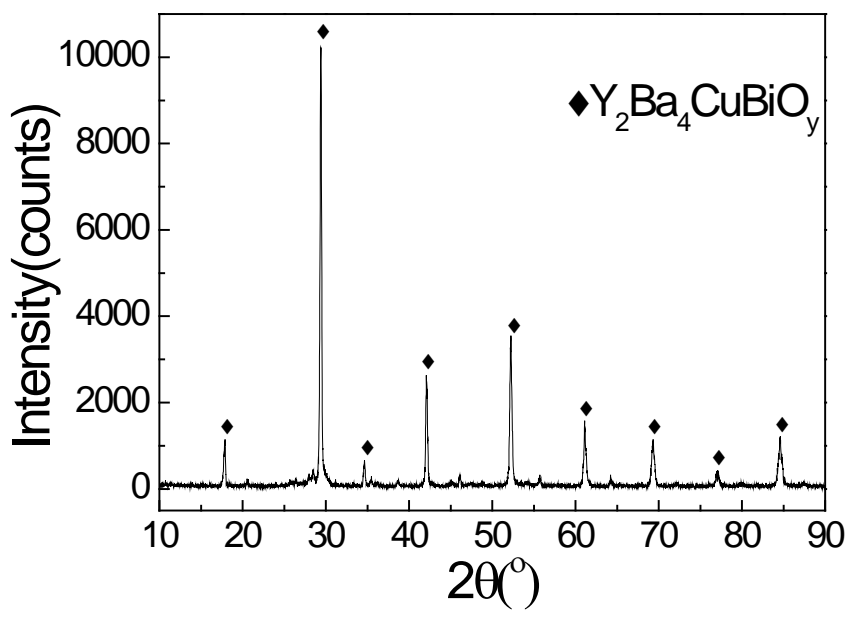

Figure 1. The XRD pattern of YBi2411 powder.

phase arising except the YBi2411 phase. This is in agreement with reference [17], which means the YBi2411 phase is of an isostructural, double perovskite cubic crystallographic structure.

\subsection{Different Ratio YBi2411 Additions to Y211}

The solid phase pellets were made by a well mixed powders of different ratio YBi2411 and Y211 particles. The ratio is in a weight percent of YBi2411:Y211 = $x:(100-x)$ (where $x=0.5,2,3$, and 5, units: wt\%) (corresponding samples are simply noted for $a_{1}, a_{2}, a_{3}$ and $a_{4}$ ), and the diameter of the solid phase pellets is pressed into $20 \mathrm{~mm}$. The liquid phase pellets were prepared from a well mixed powders of $\mathrm{Y} 123$ and $\mathrm{Ba}_{3} \mathrm{Cu}_{5} \mathrm{O}_{8}$ (Y035) powders ( $\mathrm{Y} 035$ was mixed by the powders of $\mathrm{Y} 011$ and $\mathrm{CuO}$ in a molar ratio of $\mathrm{Y} 011: \mathrm{CuO}=3: 2$ ), which were in a molar ratio of Y123:Y035 = 1:1. For all the samples, the liquid phase pellets is of $20 \mathrm{~mm}$ in diameter. Furthermore, we pressed $\mathrm{Y}_{2} \mathrm{O}_{3}$ powder into a plate of thickness of 2 $\mathrm{mm}$ to support the liquid phase at elevated temperature. And then the samples were put in a self-designed tube furnace with appropriate temperature gradient which can effectively prevent the random nucleation of YBCO grains at the edges of samples. Figure 2 shows the schematic diagram of a YBCO bulk sample before and after TSIG process. Each precursor sample is consist of two cylindrical pellets and layered up together along their coaxial line. The top one (green one) is the solid phase pellet, the next one (blank one) is the liquid source, and the third one (white one) is the $\mathrm{Y}_{2} \mathrm{O}_{3}$ pellet, as shown in the Figure 2(a). The precursor sample, with a NdBCO crystal seed which was made by self placed at the center of the top surface of the solid phase pellet, was heated up to $1045^{\circ} \mathrm{C}$ and held for $2 \mathrm{~h}$, then cooled to $1020^{\circ} \mathrm{C}$ rapidly, after that, the sample was cooled to $970^{\circ} \mathrm{C}$ at a rate of $0.2^{\circ} \mathrm{C}-1{ }^{\circ} \mathrm{C} / \mathrm{h}$, later the sample was cooled to room temperature at a rate of $120^{\circ} \mathrm{C} / \mathrm{h}$. Finally, the as grown samples were annealed in flowing oxygen for $200 \mathrm{~h}$ at temperatures ranging from $500^{\circ} \mathrm{C}$ to $410^{\circ} \mathrm{C}$, so that the as grown single-domain $\mathrm{YBCO}$ bulks could be of superconducting properties [16]. 


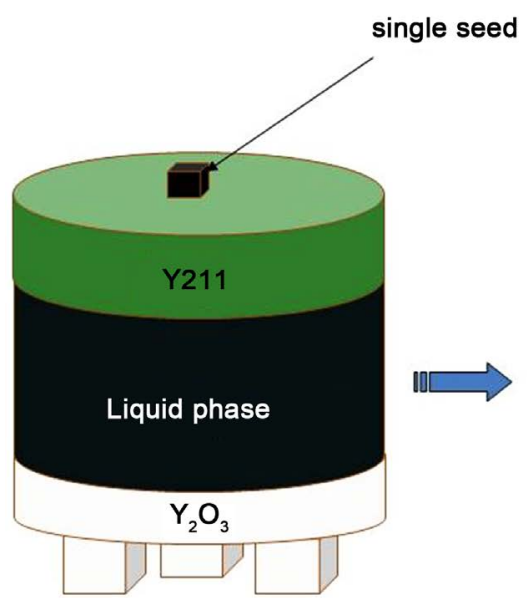

(a)

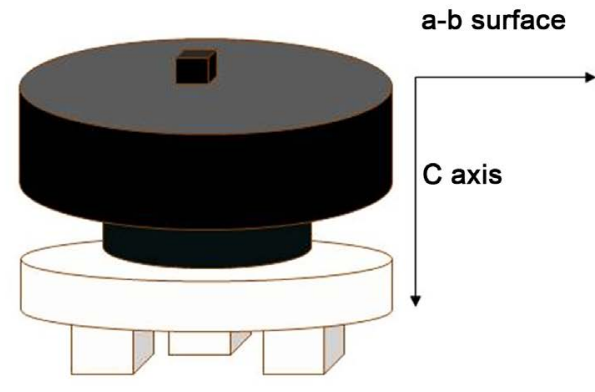

(b)

Figure 2. The schematic diagram of the sample (a: before TSIG process; b: after TSIG process). (a) Before TSIG process; (b) After TSIG process.

\section{2. $\mathrm{CeO}_{2}$ Additions to Y211 with Different Ratio YBi2411}

From the references [18] [19], it is found that the $\mathrm{CeO}_{2}$ particles doping can refine the size of $\mathrm{Y} 211$ particles effectively. And the optimum ratio of $\mathrm{CeO}_{2}$ particles doping is from $0.5 \mathrm{wt} \%$ to $2 \mathrm{wt} \%$, so $1 \mathrm{wt} \%$ of $\mathrm{CeO}_{2}$ was added to the mixture of the solid phase. The ratio of the solid phase is in a weight percent of YBi2411:Y211: $\mathrm{CeO}_{2}=x:(100-x): 1$ (also where $x=0.5,2,3$, and 5, units: wt\%) (corresponding samples are simply noted for $b_{1}, b_{2}, b_{3}$ and $b_{4}$ ). The liquid phase pellets were also mixed by the powders of Y123 and Y035 in a malor of Y123:Y035 $=1: 1$. Next assembly way of all the pellets is as the same as described in 2.1 section, as shown in Figure 2(a). Finally, the precursor samples were heated and annealed in flowing oxygen, which could be the YBCO bulk superconductors.

Then the magnetic levitation force of all the YBCO bulks was measured in the self-designed magnetic levitation force device [20]. And meanwhile, a scanning electron microscope (SEM) was used to observe microstructure of the YBCO bulk superconductors.

\section{Results and Discussion}

Figure 3 is the top view morphology of the YBCO superconductors fabricated by TSIG technique. As we can see from the samples of $a_{1}, b_{1}, c_{1}$ and $d_{1}$ in Figure 3 , the morphology of samples indicates that YBCO crystal can grow from the $\mathrm{NdBCO}$ seeds for the samples and form as a single-domain of the whole samples surface except a tiny flaw. We can found that the optimum morphology is as sample $c_{1}$, the ratio is $3 \mathrm{wt} \%$, which has not the random nucleated and is the best single-domain one.

As we can see from the samples of $a_{2}, b_{2}, c_{2}$ and $d_{2}$, which are doped with 1 wt $\% \mathrm{CeO}_{2}$ and different ratio of YBi2411, the surface macrostructure of samples is indicated that the shape of the grown crystal is different for the samples with 


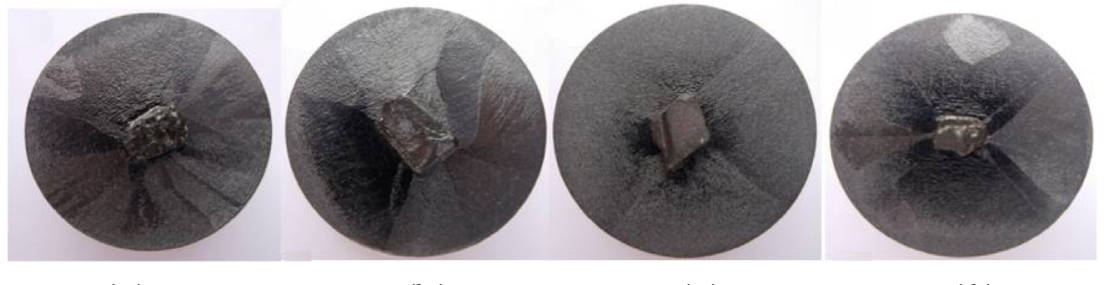

$\left(\mathrm{a}_{1}\right)$

$\left(b_{1}\right)$

$\left(c_{1}\right)$

$\left(\mathrm{d}_{1}\right)$

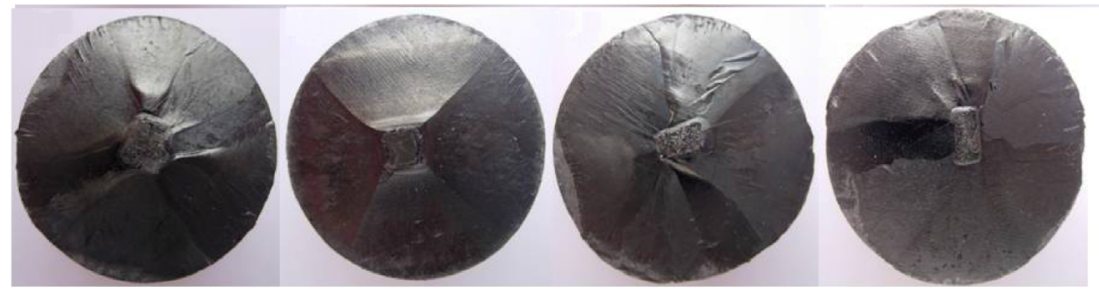

$\left(\mathrm{a}_{2}\right)$

$\left(b_{2}\right)$

$\left(\mathrm{c}_{2}\right)$

$\left(d_{2}\right)$

Figure 3. Top view of the YBCO bulk superconductors. (The above four samples are fabricated by different ratio YBi2411 additions, $\left(a_{1}\right) x=0.5 w t \%,\left(b_{1}\right) x=2 w t \%,\left(c_{1}\right) x=3$ wt $\%,\left(d_{1}\right) x=5$ wt $\%$; the below four samples are fabricated by $1 \mathrm{wt} \% \mathrm{CeO}_{2}$ with different YBi2411 addtions, $\left(a_{1}\right) x=0.5 w t \%,\left(b_{1}\right) x=2 w t \%,\left(c_{1}\right) x=3 w t \%,\left(d_{1}\right) x=5 w t \%$.)

different YBi2411 additions. When $x \leq 2 \mathrm{wt} \%$, the YBCO crystal can grow up to the whole samples and forms as a single-domain, as shown in Figure $3\left(\mathrm{a}_{2}\right)$ and Figure $3\left(b_{2}\right)$; when $x>2 \mathrm{wt} \%$, the YBCO crystal cannot grow up to the single domainand there are some random nucleated $\mathrm{YBCO}$ grains at the edge of the samples, as shown in Figure $3\left(c_{2}\right)$ and Figure $3\left(d_{2}\right)$. And from these four figures, the optimum YBi2411 nanoparticles doping is $2 \mathrm{wt} \%$. So we can know that the best ration of the YBi2411 nanoparticles additions is reduced compared with the samples without $\mathrm{CeO}_{2}$ doping.

Figure 4 is the levitation force-distance $(\mathrm{Z})$, curves between the magnet and the samples of YBCO superconductors, which were measured at $77 \mathrm{~K}$ under zero-field cooling state. It is found from Figure 4(a) that the levitation force is much different for the samples with different YBi2411 particles additions, the levitation force increases from $7.1 \mathrm{~N}$ to $17.8 \mathrm{~N}$ as the YBi2411 additions $x$ increases from $0.5 \mathrm{wt} \%$ to $3 \mathrm{wt} \%$, and then it decreases from $17.8 \mathrm{~N}$ to $11.6 \mathrm{~N}$ as the YBi2411 content $x$ increases from $3 \mathrm{wt} \%$ to $5 \mathrm{wt} \%$. The largest levitation force is obtained in the sample with about $3 \mathrm{wt} \%$ YBi2411 nanoparticles additions. It implies that the reasonable particles of YBi2411 additions are helpful for us to improve the levitation force of the YBCO bulk superconductor. Similarly, the samples, which are doped to different YBi2411 particles with $1 \mathrm{wt} \% \mathrm{CeO}_{2}$ additions, levitation force of which were also measured. From Figure 4(b), we can see that the largest levitation force is about $15 \mathrm{~N}$, and the force is obtained in the sample with $2 \mathrm{wt} \%$ YBi2411 additions, which is much larger than the sample (2 wt\% YBi2411 nanoparticles additon) without $\mathrm{CeO}_{2}$ doping. The comparison diagram was exhibited as shown in Figure 5. From this figure, it is found that when YBi2411 content $x$ is $0.5 \mathrm{wt} \%$ and $2 \mathrm{wt} \%$, the levitation force of the samples which were doped $1 \mathrm{wt} \% \mathrm{CeO}_{2}$ are much larger than those without $\mathrm{CeO}_{2}$. 


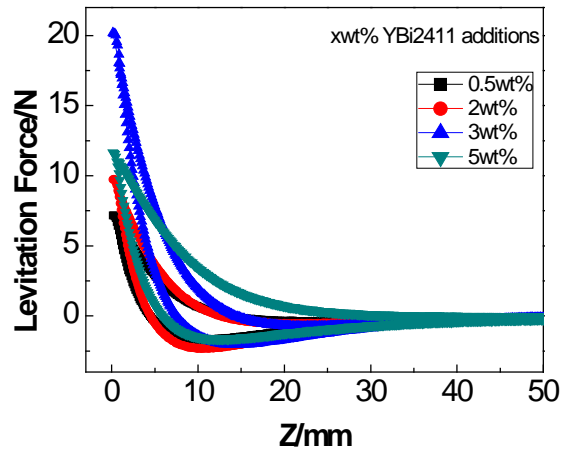

(a)

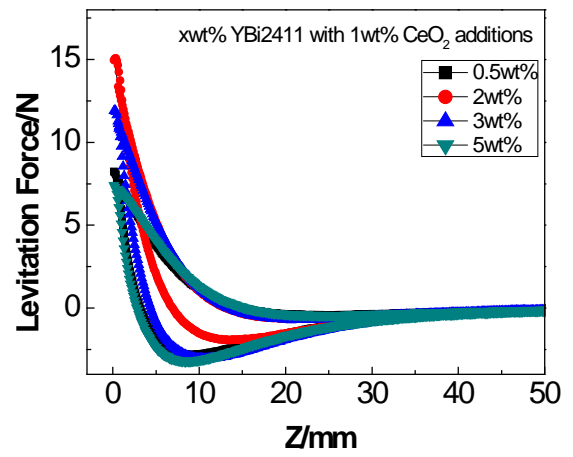

(b)

Figure 4. Levitation force of the samples ((a): different additions of YBi2411 to Y211; (b): different ratios of $\mathrm{YBi} 2411$ with $1 \mathrm{wt} \% \mathrm{CeO}_{2}$ to $\left.\mathrm{Y} 211\right)$.

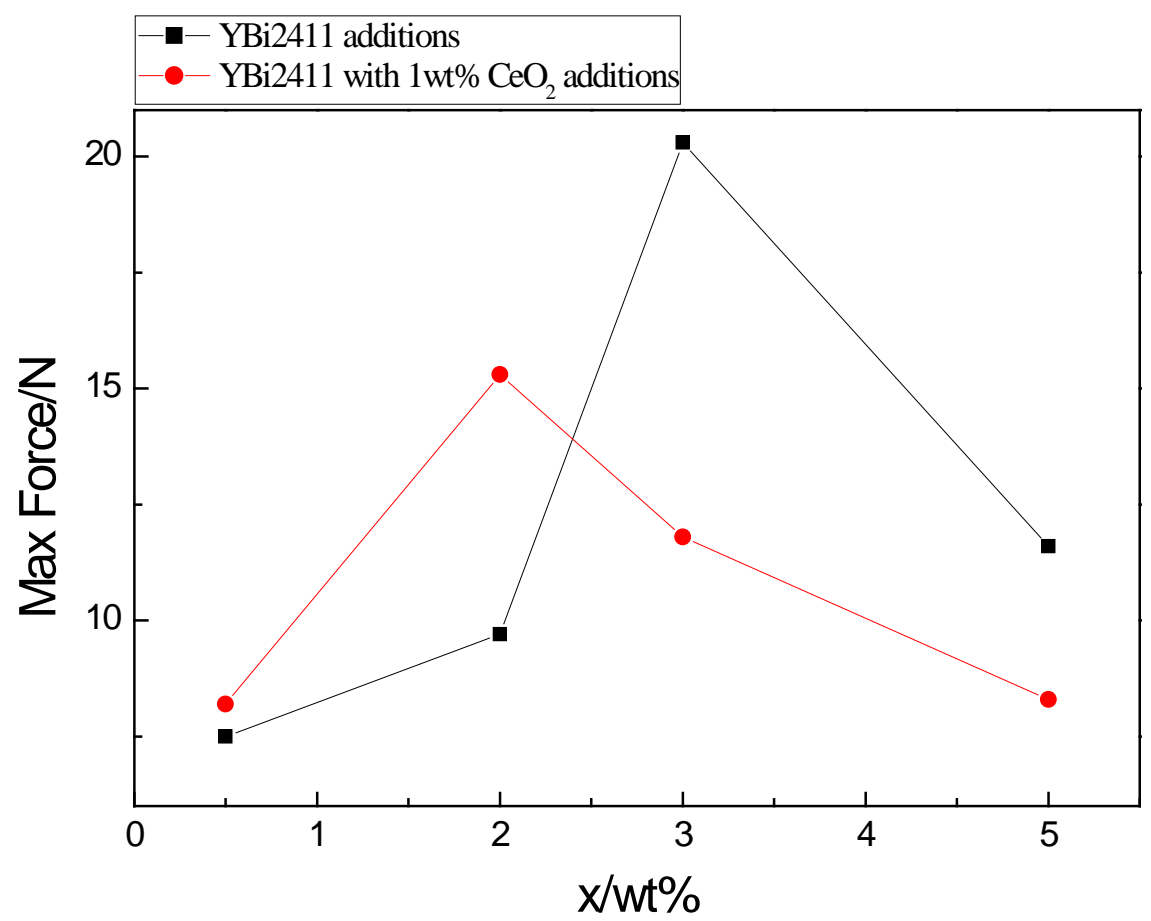

Figure 5. The comparison diagram of the max levitation force.

This is due to that the $\mathrm{CeO}_{2}$ particles doping can effectively refine the size of Y211, which added more flux pinning center into the superconductor samples. Consequently, the levitation force can be increased effectively. On the contrary, the levitation force is much different when $x$ is increased from $3 \mathrm{wt} \%$ to $5 \mathrm{wt} \%$. The levitation force of the samples which were doped $1 \mathrm{wt} \% \mathrm{CeO}_{2}$ are rapidly down compared to those without $\mathrm{CeO}_{2}$, and the force were fallen by half. For instance, the levitation force is about $20 \mathrm{~N}$ when $3 \mathrm{wt} \%$ YBi2411 doped, but it is rapidly down to $7 \mathrm{~N}$ when $3 \mathrm{wt} \% \mathrm{YBi} 2411$ with $1 \mathrm{wt} \% \mathrm{CeO}_{2}$ doping. This result indicates that, $1 \mathrm{wt} \% \mathrm{CeO}_{2}$ doping can make reduction the amounts of $\mathrm{YBi} 2411$ particles for fabricating high performance YBCO supercondcutors.

Figure 6 shows the Quanta 200 scanning electron micrographs (SEM) of the 

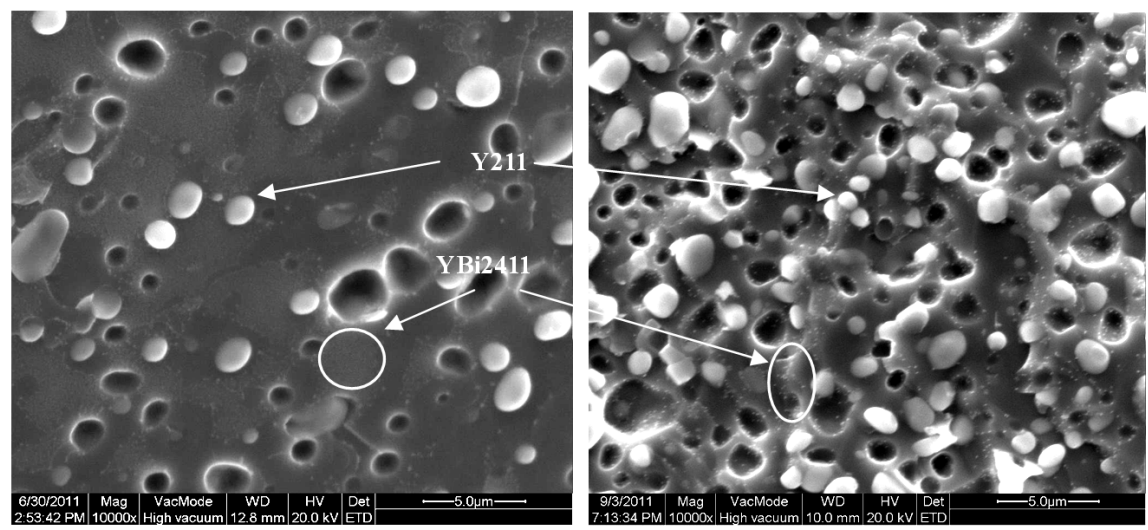

Figure 6. Scanning electron micrographs at a/b plane of the YBCO bulks. (a) 3 wt\% YBi2411 additions to Y211; (b) $1 \mathrm{wt} \% \mathrm{CeO}_{2}$ with $2 \mathrm{wt} \%$ YBi2411 additions to Y211.

cross-sections of the YBCO bulk samples. As we can see from these two figures, there are two kinds of particles distributed in the YBCO matrix, one is Y211 light grey particle, which is distributed in the samples, and the size of these particles is in the range from $2.0 \mu \mathrm{m}$ to $5.0 \mu \mathrm{m}$. The other is the YBi2411 particle, and it is also near uniformly distributed in the samples. Furthermore, the width of the YBi2411 particles is in the range from $100 \mathrm{~nm}$ to $500 \mathrm{~nm}$, which is indeed much smaller than that of the Y211 particles. From these figures, it is clear that the YBi2411 phase remains stable during the solidification process, but the size of these particles is quite different. The average size of the YBi2411 particles is nearly about $300-500 \mathrm{~nm}$ when $3 \mathrm{wt} \%$ YBi2411 additions, which is not uniformly distributed in the sample, as shown in Figure 6(a), and it is found that YBi2411 particles will be accumulated together and formed the platelike structure in some regional. On the contrary, it is different when $1 \mathrm{wt} \% \mathrm{CeO}_{2}$ doped. It is found that the sizes of the YBi2411 particles are much smaller than the former one, and on average it is about $100 \mathrm{~nm}$. As shown in Figure 6(b), not only the distribution of the YBi2411 particles is scattered, but also the Y211 particles is. Consequently, the equably dispersed of the YBi2411 particles are highly increased the total area of the YBi2411/Y123 superconductivity interface, and also greatly increased the area of YBCO superconductors, which lead to an addition of magnetic flux pinning force and levitation force of the samples with $\mathrm{CeO}_{2}$ addition. This is in agreement with the levitation force as shown in Figure 5.

\section{Conclusion}

Single domain YBCO superconductor bulks have been fabricated by TSIG process with different ratio YBi2411 nanoscale particles additions. We have investigated the effect of the single domain YBCO bulk superconductors by TSIG process. It is found that $2 \mathrm{wt} \% \mathrm{YBi} 2411$ particles with $1 \mathrm{wt} \% \mathrm{CeO}_{2}$ doping can fabricate the larger levitation force of YBCO superconductor bulk, which has the significant influence to the development of application of high temperature superconductor REBCO in the future. 


\section{Acknowledgements}

This work is supported by the National Natural Science Foundation of China (Grant No. 11647050), and the Undergraduate Innovation and Entrepreneurship Training Program of Xi'an Aeronautical University (Grant No. DCX2016015).

\section{References}

[1] Yang, W.M., Zhou, L., Feng, Y., Zhang, P.X., Wu, M.Z., Gawalek, W. and Gornert, P. (1998) The Effect of Excess $\mathrm{Y}_{2} \mathrm{O}_{3}$ Addition on the Levitation Force of Melt Processed YBCO Bulk Superconductors. Physica C: Superconductivity, 305, 269-274. https://doi.org/10.1016/S0921-4534(98)00339-6

[2] Li, G.Z., Yang, W.M., Cheng, X.F., Guo, X.D. and Fan, J. (2009) A Modified TSIG Technique for Simplifying the Fabrication Process of Single-Domain GdBCO Bulks with a New Kind of Liquid Source. Journal of Materials Science, 44, 6423-6426. ttps://doi.org/10.1007/s10853-009-3887-8

[3] Hari Babu, N., Cheng, L., Iida, K. and Cardwell, D.A. (2006) Nano-Composite Single Grain $\mathrm{YBa}_{2} \mathrm{Cu}_{3} \mathrm{O}_{7-\delta} / \mathrm{Y}_{2} \mathrm{Ba}_{4} \mathrm{CuBiO}_{\mathrm{y}}$ Bulk Superconductors. Journal of Physics: Conference Series, 43. https://doi.org/10.1088/1742-6596/43/1/094

[4] Meslin, S. and Noudem, J.G. (2004) Infiltration and Top Seeded Grown MonoDomain $\mathrm{YBa}_{2} \mathrm{Cu}_{3} \mathrm{O}_{7-\mathrm{x}}$ Bulk Superconductor. Superconductor Science and Technology, 17. https://doi.org/10.1088/0953-2048/17/11/014

[5] Iida, K., Hari Babu, N., Shi, Y. and Cardwell, D.A. (2006) Seeded Infiltration and Growth of Single-Domain GdBaCuO Bulk Superconductors Using a Generic Seed Crystal. Superconductor Science and Technology, 19. https://doi.org/10.1088/0953-2048/19/7/S11

[6] Hari Babu, N., Iida, K., Shi, Y. and Cardwell, D.A. (2005) Fabrication of High Performance Light Rare Earth Based Single-Grain Superconductors in Air. Applied Physics Letters, 87, 20.

[7] Yang, P.T., Yang, W.M., Abula, Y., Su, X.Q. and Zhang, L.L. (2017) Effect of Li Doping on the Superconducting Properties of Single Domain GdBCO Bulks Fabricated by the Top-Seeded Infiltration and Growth Process. Ceramic International, 43,3 .

[8] Noudem, J.G., Meslin, S., Horvath, D., Harnois, C., Chateigner, D., Eve, S., Gomina, M., Chaud, X. and Murakami, M. (2007) Fabrication of Textured YBCO Bulks with Artificial Holes. Physica C: Superconductivity and its Applications, 463-465, 301307. https://doi.org/10.1016/j.physc.2007.03.503

[9] Ouerghi, A., Moutalbi, N., Noudem, J.G. and M'chirgui (2017) A Single-Grain YBCO Bulk Superconductor through the Infiltration-Growth Process. Physica $C$ : Superconductivity and its Applications, 534, 37-44. https://doi.org/10.1016/j.physc.2017.01.003

[10] Izumi, T., Nakamura, Y. and Shiohara, Y. (1993) Doping Effects on Coarsening of $\mathrm{Y}_{2} \mathrm{BaCuO}_{5}$ Phase in Liquid. Journal of Materials Research, 8, 1240-1246. https://doi.org/10.1557/JMR.1993.1240

[11] Ogawa, N., Hirabayashi, I. and Tanaka, S. (1991) Preparation of a High J yBCO $^{\circ}$ Bulk Superconductor by the Platinum Doped Melt Growth Method. Physic C, 177, 101-105. https://doi.org/10.1016/0921-4534(91)90304-H

[12] Athur, S.P., Selvamanickam, V., Balachandran, U. and Salama, K. (1996) Study of Growth Kinetics in Melt-Textured $\mathrm{YBa}_{2} \mathrm{Cu}_{3} \mathrm{O}_{7-\mathrm{x}}$. Journal of Materials Research, 11, 2976-2989. https://doi.org/10.1557/JMR.1996.0379 
[13] Foltyn, S.R., Civale, L., Mannus-Driscol, J.L., Jia, Q.X., Maiorov, B., Wang, H. and Maley, M. (2007) Materials Science Challenges for High-Temperature Superconducting Wire. Nature Materials, 6, 631-642. https://doi.org/10.1038/nmat1989

[14] Cardwell, D.A. and Hari Babu, N. (2006) Processing and Properties of Single Grain (RE)-Ba-Cu-O Bulk Superconductors. Physica C: Superconductivity and Its Applications, 445-448, 1-7. https://doi.org/10.1016/j.physc.2006.03.065

[15] Hari Babu, N., Lo, W., Cardwell, D.A. and Shi, Y.H. (1999) The Effect of Undercooling and Nd422 Phase Content on the Nucleation of Large Nd-Ba-Cu-O Grains Fabricated by Top-Seeded Melt Processing. Journal of Materials Research, 14, 38593863.

[16] Wang, M., Yang, W.M., Wang, M.Z. and Wang, X.J. (2013) Effect of $\mathrm{Y}_{2} \mathrm{Ba}_{4} \mathrm{CuBiO}_{\mathrm{y}}$ Nanoparticles Doping on the Levitation Force of Single-Domain YBCO Bulk Superconductor by Top-Seeded Infiltration Process. Journal of Superconductivity and Novel Magnetism, 26, 3221-3224. https://doi.org/10.1007/s10948-013-2179-3

[17] Hari Babu, N., Iida, K. and Cardwell, D.A. (2007) Flux Pinning in Melt-Processed Nanocomposite Single-Grain Superconductors. Superconductor Science and Technology, 20, S141-S146. https://doi.org/10.1088/0953-2048/20/9/S05

[18] Harnois, C.L., Laffez, I.M. and Desgardion, G. (2000) Texturing Process, Superconducting and Mechanical Properties of Ag-Doped Top-Seeded Melt-Grown YBCO Pellets. Physica C: Superconductivity, 341-348, 2439-2440.

[19] Kim, Y., No, K., Han, Y.H., Kim, C.J., Jun, B.H., Lee, D.Y., Youn, J.S. and Sung, T.H. (2011) Interaction Mediated by Size Differences between $\mathrm{YBaCuO}$ and $\mathrm{CeO}$ Particles in Melt-Textured YBCO Superconductors. Cryogenics, 51, 247-252. https://doi.org/10.1016/j.cryogenics.2010.06.005

[20] Yang, W.M., Chao, X.X., Shu, Z.B., Zhu, S.H., Wu, X.L., Bian, X.B. and Liu, P. (2006) A Levitation Force and Magnetic Field Distribution Measurement System in Three Dimensions. Physica C: Superconductivity and Its Applications, 445-448, 347-352. https://doi.org/10.1016/j.physc.2006.04.080 